\title{
Modern considerations when approaching fractured endodontic instruments - Part 2: A review of the literature and clinical techniques
}

SADJ November 2020, Vol. 75 No. 10 p564 - p574

PJ van der Vyver ${ }^{1}$, M Vorster ${ }^{2}$, CH Jonker ${ }^{3}$

\section{INTRODUCTION}

Once root canal treatment is considered, the treating clinicians must be aware of the real possibility that complications and unforeseen accidents can occur during any stage of the treatment. Complications and accidents may include instrument separation, root perforation on different levels and ledge formation.

Once complications and accidents arise during any part of the cleaning and shaping procedure, the tooth can be compromised and the prognosis can ultimately be reduced. ${ }^{1-3}$ The reduced prognosis may even extend to the final obturation and $3 D$ sealing of the root canal system. ${ }^{2,3}$

The ultimate goals of any root canal treatment are to remove or destroy micro-organisms within the root canal system, remove necrotic or infected pulp tissue and finally achieve an acceptable obturation and sealing of all root canal spaces. ${ }^{1}$ Where separation of endodontic instruments occurs it can create one of the most stressful and unpleasant situations for both the clinician and patient. The occurrence can have a "snowball" effect: a patient can perceive the incident as a treatment failure (even as clinical negligence if complications arise); final coronal restorability is compromised; and if medico-legal action is taken, conflict can arise between the treating clinician and referring dentist. ${ }^{4}$

Author affiliations:

1. Peet J van der Vyver: $B C h D, P G$ Dip Dent (Endo), PG Dip Dent (Aesthet Dent), MSc, PhD (Pret), Department of Odontology, School of Dentistry, University of Pretoria, Pretoria, South Africa. ORCID Number: 0000-0003-1951-6042

2. Martin Vorster: BChD, PG Dip Dent (Endo), MSc (Pret), Department of Odontology, School of Dentistry, University of Pretoria, Pretoria, South Africa.

ORCID Number: 0000-0003-4470-1530

3. Casper H Jonker: BChD (Pret), Dip Odont (Endo), MSc (Pret), Module of Endodontics, Department of Operative Dentistry, Sefako Magatho Health Sciences University, Ga-Rankuwa, South Africa.

ORCID Number: : 0000-0002-9110-5208

Corresponding author: Peet $\mathrm{J}$ van der Vyver

Department of Odontology, School of Dentistry, University of Pretoria,

Gauteng, South Africa.

Email: peetv@iafrica.com

Author contributions:

Peet J van der Vyver: Principal author - 33.3\%

2. Martin Vorster: Co-author $-33.3 \%$

3. Casper H Jonker: Co-author - $33.3 \%$
In the literature, instrument failures are attributed to various factors. One of these is the incorrect use of instruments; operators can ignore the techniques advocated by the manufacturers or operate instruments already fatigued by repeated use. ${ }^{5,6}$

\section{Treatment considerations}

Once a clinician is confronted with a fractured endodontic instrument, it is important to follow a structured approach. The clinician must realise that the treatment could be challenging and must also be aware of complicating factors, which may include:

1. the complexity of the root canal system.

2. the treating clinician's access to the materials, instruments and devices needed to attempt removal.

3. whether the clinician can predict the outcome of the attempt removal considering his or her experience.

4. the location, size, position and diameter of the fractured fragment. ${ }^{6,7}$

The clinician should also consider the wishes of the patient, who might decide on extraction due to financial and/or time constraints or anxiety. ${ }^{4}$ The interests of the patient are paramount. There are also a number of clinical factors to consider before treatment is attempted: 4

\section{Periodontal and restorative prognosis}

In general, periodontally compromised patients are not ideal candidates for instrument removal unless the periodontal health is stable and well maintained. Restorability also needs to be considered when periodontal breakdown is diagnosed. 4

\section{General patient factors}

An attempt to remove a fractured instrument can consume clinical chair time. A patient might be apprehensive or might have time constraints. ${ }^{4}$ On the other hand, certain medical conditions (bleeding disorders and certain medications, including bisphosphonate) may tip the balance towards removing the fractured fragment where extraction is contra-indicated. ${ }^{8}$ Treatment costs can also 
play a role, as certain cases may involve referral to a specialist endodontist or a clinician with proven experience in the field of endodontics. ${ }^{4}$

The following options can be considered once the decision is made to proceed with treatment:

\section{Leaving the fragment in situ}

This option involves incorporating the fragment into the final obturation. ${ }^{9}$ Leaving a fractured instrument in situ can be risky in cases with apical lesions as the healing will be reduced, ${ }^{2,10}$ but in selected vital cases or cases where advanced mechanical-chemical disinfection is available and the prognosis is considered more favourable, this treatment approach can be considered. ${ }^{11,12}$

\section{CASE REPORT 1}

A 23-year-old female presented with a fractured file in the mesio-lingual root canal system of her mandibular right first molar, approximately $3 \mathrm{~mm}$ from the apex of the root (Figure 1A). A CBCT scan revealed a large periapical lesion around the mesial root and a small periapical lesion around the distal root canal (Figures 1B and C).

An attempt was made to remove the fractured instrument using ultrasonics. After 40 minutes the attempt was unsuccessful, and a perforation was caused in the furcation area of the tooth. It was decided to leave the fractured file in situ and to repair the perforation with MTA (Figure 1D). The other three root canal systems were negotiated and prepared with a WaveOne Gold Primary file (Dentsply Sirona).

Root canal obturation of the other three canals was done with Primary WaveOne gutta percha cones (Dentsply Sirona) and pulp canal sealer (Kerr) using the warm vertical condensation technique. A two-year postoperative follow-up periapical radiograph (Figure 1E) and CBCT scan (Figures $1 \mathrm{~F}$ and $\mathrm{G}$ ) revealed good healing of the periapical pathology.

\section{Bypass the fragment}

In general, space can be created by inserting small hand files between the fragment and the root canal. Although a time-consuming and labour-intensive exercise, full working length negotiation can be achieved.

There is also a good possibility that the fragment can be loosened and removed during bypassing. In selected cases where no movement is achieved, the fragment can be left in situ and incorporated to form an integrated part of the final obturation. ${ }^{9}$

\section{CASE REPORT 2}

A 45-year-old female presented with a fractured file in the mesio-buccal root canal of her mandibular right
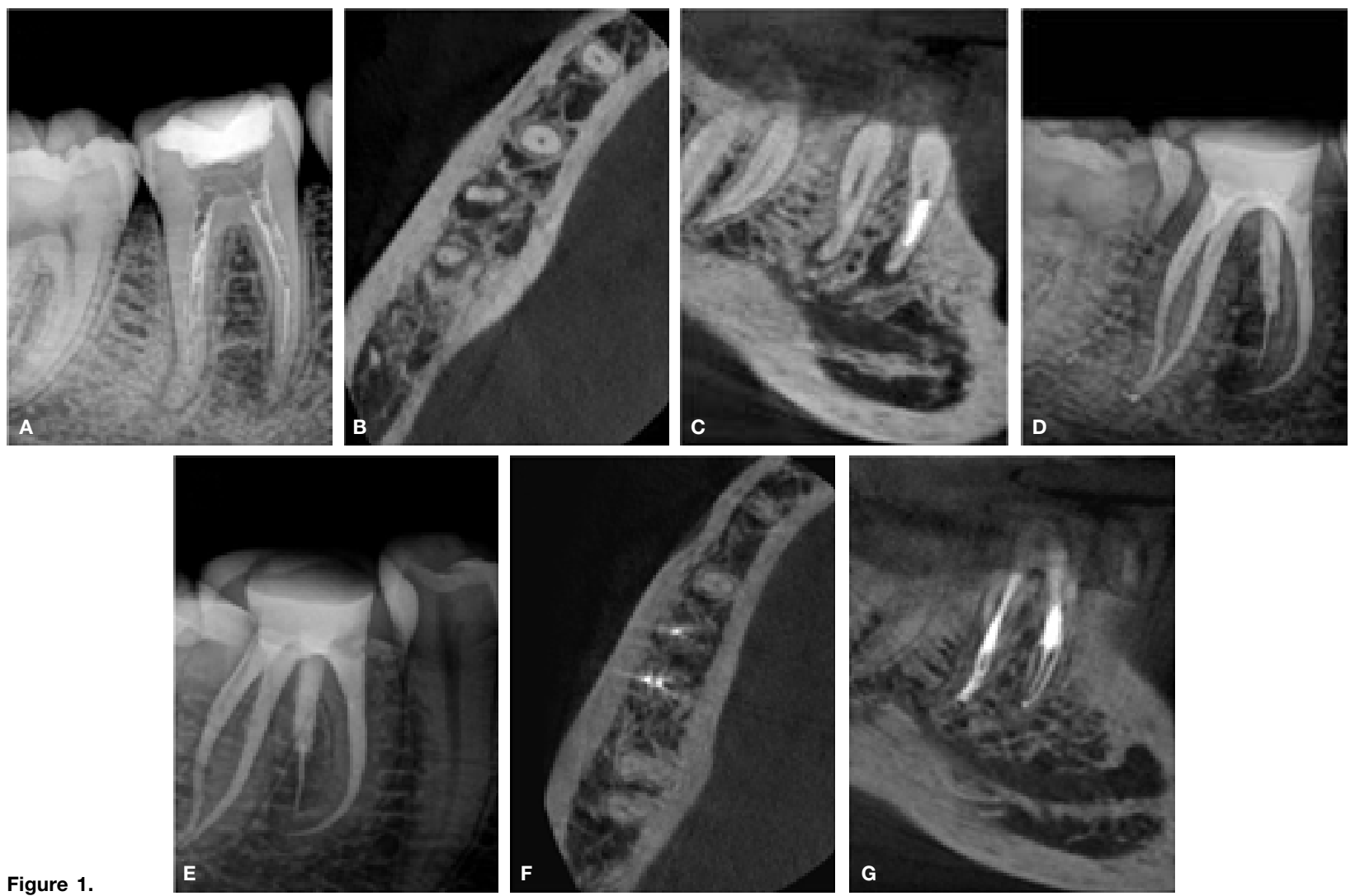

Figure 1.

D. Repair of perforation with MTA and fractured file left in situ.

A. Pre-operative periapical radiograph of the mandibular right
with a fractured file in the mesio-lingual root canal system.

E. A two-year post-operative periapical radiograph.

B. Axial view on CBCT demonstrating a large periapical lesion around the mesial root and a small periapical lesion around the distal root.

C. Sagittal view on CBCT showing a large periapical lesion around the mesial root and a small periapical lesion around the distal root.

F. Axial view on a two-year postoperative CBCT showing healing of periapical pathology.

G. Sagittal view on a two-year postoperative CBCT demonstrating healing of periapical pathology. 
second molar (Figure 2A). It was a fragment of approximately $7 \mathrm{~mm}$, located at the apex of the root canal system. After an unsuccessful attempt by her general dentist to remove the fragment with ultrasonic instruments, she was referred for further management.

At the time of treatment it was decided to attempt to bypass the file because (1) examination of a CBCT scan revealed that the fragment was located beyond the maximum curvature of the root canal system; (2) under high microscope magnification the coronal aspect of the fractured fragment was not visible, even after coronal enlargement of the root canal system by her general dentist.

A size 08 C+ (Dentsply Sirona) and 08 K-File (Dentsply Sirona) were precurved and used alternately to bypass the fractured fragment (Figure 2B). The new glide path next to the fractured instrument was carefully enlarged with a size $10 \mathrm{~K}$-File, followed by a size 12 Profinder (Dentsply Sirona) and a ProGlider rotary glide path instrument (Dentsply Sirona) used in a manual motion.

Canal preparation was completed with the TruNatomy Prime file in the mesial root canal systems and the TruNatomy Medium file (Dentsply Sirona) in the distal root canal system. The fit of two Prime and one Medium TruNatomy gutta percha cones (Dentsply Sirona) was verified radiographically (Figure $2 \mathrm{C}$ ) before the canals were obturated with $\mathrm{AH}$ Plus root canal cement (Dentsply Sirona) using the Gutta Smart Obturation System (Dentsply Sirona) (Figure 2D).

\section{Removal of the fractured instrument}

Attempting to remove a fractured instrument can be a very challenging exercise. ${ }^{13}$ Hulsmann ${ }^{14}$ states that there is no standard method of removing fractured instruments and that a number of approaches can be followed.

It must be emphasised that no matter what technique is used, proper vision, illumination and magnification play a crucial role when attempting retrieval. ${ }^{15}$ The Dental Operating Microscope (DOM) increases direct visualisation of the instrument fragment where normal vision is inadequate. ${ }^{16}$ A study by Nevares et al. ${ }^{17}$ concludes that the success rate in removing or bypassing frac-
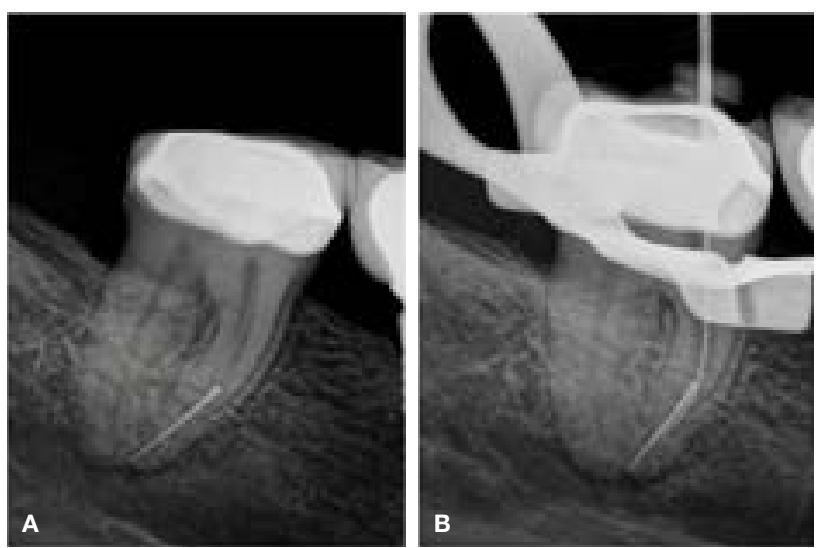

Figure 2.

A. Pre-operative periapical radiograph of a mandibular right second molar with a fractured instrument in the mesio-buccal root canal system.

B. Fractured file bypassed with a size $08 \mathrm{~K}$-File. tured instruments is doubled when the fragment is visible under the DOM.

In recent years several new techniques and devices used in the removal of separated instruments have been introduced in the market. It is important to note that the clinician's skills and experience are crucial aspects when deciding which technique is best suited for separated instrument removal. Referral to a clinician or specialist with more experience in instrument retrieval might prove a better alternative in cases where the clinician's skill is lacking. Some widely used techniques and some more recent promising techniques are discussed below.

\section{i. Ultrasonics}

Ultrasonics is probably the most widely used technique in endodontics in the removal of separated instruments. A Gates Glidden drill (GG) no.1 (0.50 mm), GG no.2
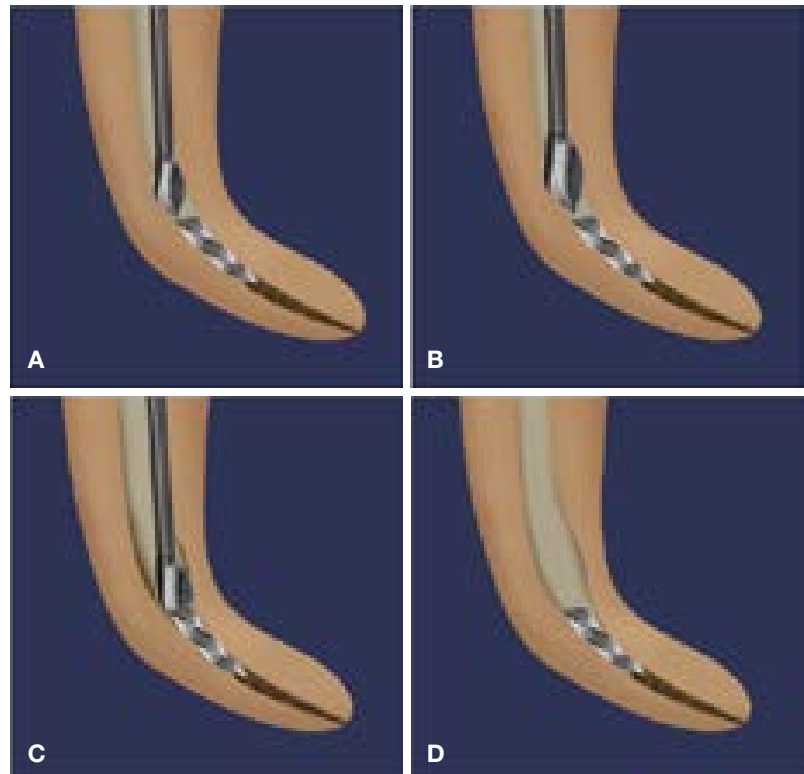

Figure 3. (Adapted from Terauchi, $2012^{37}$ )

A. GG drill no. 1 or GG no. 2 is taken to the depth of the separated instrument to create radicular access to the obstruction.

B. GG drill no. 2 or GG no. 3 is taken to the depth of the separated instrument to create further radicular access to the obstruction.

C. Creation of a staging platform with a modified GG bur.

D. Completed staging platform that allows enough space lateral to the broken file segment to initiate trephining procedures.

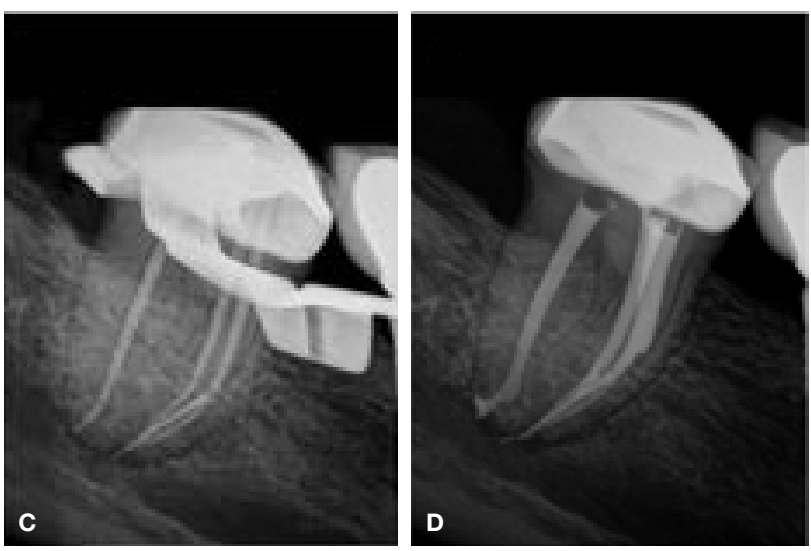

C. TruNatomy Prime gutta percha points fitted after preparation with the TruNatomy Prime file.

D. Postoperative periapical radiograph after obturation. 
$(0.70 \mathrm{~mm})$ or $\mathrm{GG}$ no.3 $(0.90 \mathrm{~mm})$ can usually be taken to the depth of the separated instrument to create radicular access to the obstruction (Figures $3 \mathrm{~A}$ and $\mathrm{B}$ ). In addition, it is recommended to create a staging platform with a modified GG bur (Figure 3C). A GG drill whose maximum cross-sectional diameter is slightly larger than the visible instrument is modified by cutting it perpendicular to its long axis at its maximum crosssectional diameter with a diamond bur. The staging platform (Figure 3D) allows enough space lateral to the broken file segment to initiate trephining procedures with ultrasonic instruments. ${ }^{18-21}$

The clinician should be able to see the separated instrument with the use of magnification and illumination. ${ }^{22}$ An ultrasonic tip is used to create a gutter around the fractured instrument before dislodging the separated portion using ultrasonic agitation. For a better view, canals should be irrigated and dried before introducing the ultrasonic tips into the canal. Ultrasonic instruments
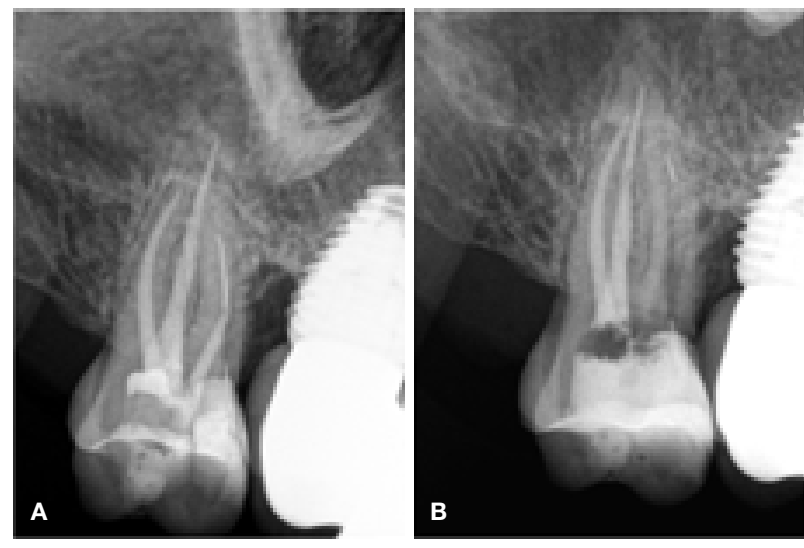

Figure 4.

A. Pre-operative periapical radiograph of a symptomatic maxillary second molar with a fractured instrument in the mesio-buccal root canal system.

B. During an attempt to remove the fractured instrument with ultrasonics a secondary file fracture was caused, leaving the retained fragment much deeper in the root canal system. of different tip sizes and diameters that can be adapted to different sections of the root canal are commercially available. ${ }^{11}$ Magnification is essential when using these

tips, as blind trephining might lead to undesired removal of dentine along the root canal walls. Nagai et al. ${ }^{23}$ report a $67 \%$ success rate in removing separated instrument fragments using ultrasonics; other success rates of $88 \%{ }^{24}$ and even as high as $95 \%$ have been reported. ${ }^{25}$

It is important to note that the use of ultrasonics can sometimes cause secondary fracture of the separated fragment (Figures 4A and B). In a study by Ward et al., ${ }^{26}$ where ultrasonics was used to remove separated files from simulated canals and extracted teeth, they observe that a portion of the instrument would occasionally break off from the original fragment, leaving a shorter fragment behind. In general, shorter fragments are more difficult to remove than longer ones, and the retrieval rates are low for fragments that are located apical to the canal curvature. ${ }^{27-29}$

Ultrasonic vibration also sometimes pushes the separated file deeper down the canal, and aggressive or incorrect use of ultrasonics sometimes results in perforation of the root canal. Another disadvantage is that the prolonged use of ultrasonics can result in a temperature rise on the root surface. ${ }^{30-32}$ According to Sweatman et al., ${ }^{33}$ if the temperature increase on the root surface goes beyond $10^{\circ} \mathrm{C}$ the periodontal tissues can be seriously damaged, especially when the ultrasonic energy is used without coolant to enhance the view. ${ }^{20,23,26,29,34}$

The increase in temperature depends on the duration of activation, type of ultrasonic tip, power settings, and the use of coolants such as water and air. ${ }^{27,35} \mathrm{~A}$ study by Madarati, Qualtrough and Watts ${ }^{36}$ shows that ultrasonic activation with constant air flow as coolant did keep the temperature rise significantly lower than activation without the air flow during file removal attempts.
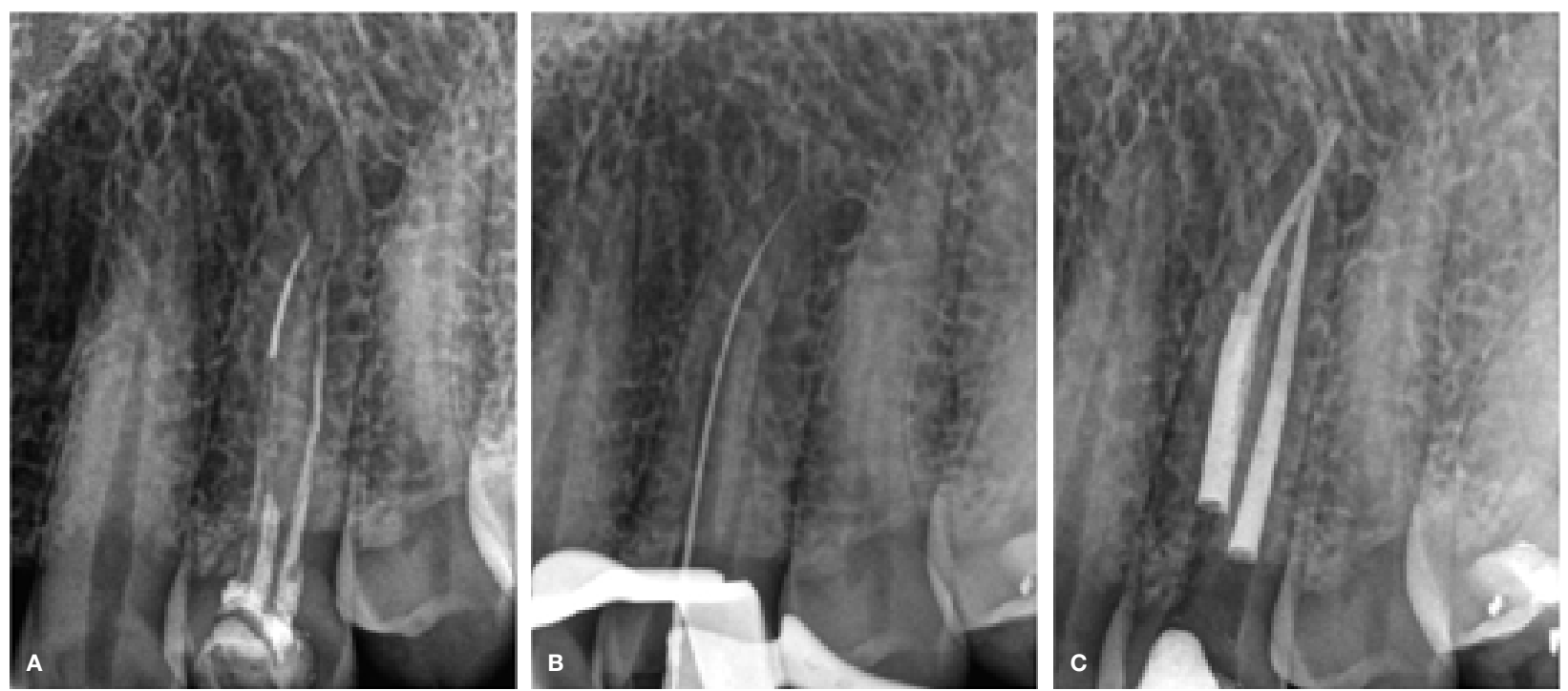

Figure 5.

A. Pre-operative periapical radiograph of maxillary left first premolar with poor root canal treatment and a fractured instrument in the buccal root canal system.
B. Fractured file was removed with ultrasonics and the canal negotiated to working length.

C. Postoperative periapical radiograph after obturation of the root canal systems. 
They also demonstrate that an increase in temperature generated by ultrasonics within the canal might be several times higher than the readings recorded on the external root surface. ${ }^{36}$

\section{CASE REPORT 3}

A 38-year-old male presented with a history of pain and discomfort on his maxillary left first premolar. Radiographic examination revealed a previous root canal treatment on the tooth with a fractured file in the buccal root canal and inadequate root canal preparation and obturation in the palatal root canal (Figure 5A).

The existing gutta percha was removed from the root canal systems with Endosolv E (Septodont) and a size 15 Hedstrom file (Dentsply Sirona). A staging platform was prepared with a size 2 GG bur to create straightline access into the root canal system and to expose the coronal aspect of the fractured fragment.

A size 15 Endosonare file (Dentsply Sirona) mounted in a U-File holder (Endo Kit E12, NSK) and attached to a Satelec P-5 ultrasonic scaler (Satelec) was used to trough around the fragment in a anticlockwise direction until the file was dislodged from the root canal.

The canals were negotiated (Figure 5B), glide paths were prepared and root canal preparation was completed before the root canals were irrigated with EDTA and heated $3.5 \%$ sodium hypochlorite before obturation (Figure 5C).

\section{ii. Zumax Broken Instrument Removal Kit (Zumax Medical)}

As with the ultrasonic technique, the first step in using the Zumax Broken Instrument Removal Kit (Zumax Medical) is creating radicular access and a staging platform using conventional and modified GG drills.

The next step is selecting one of the three trephine burs whose maximum cross-sectional diameter is slightly larger than the visible coronal aspect of the fractured instrument. The trephine is operated at a speed of $600 \mathrm{rpm}$ in a contra-angle hand piece to remove a small amount of dentine around the fractured segment in order to expose the coronal aspect of the fragment for about $2.5-3 \mathrm{~mm}$. The trephine burs are available in sizes $0.8,1.0$ and $1.2 \mathrm{~mm}$ in diameter (Figure 6).

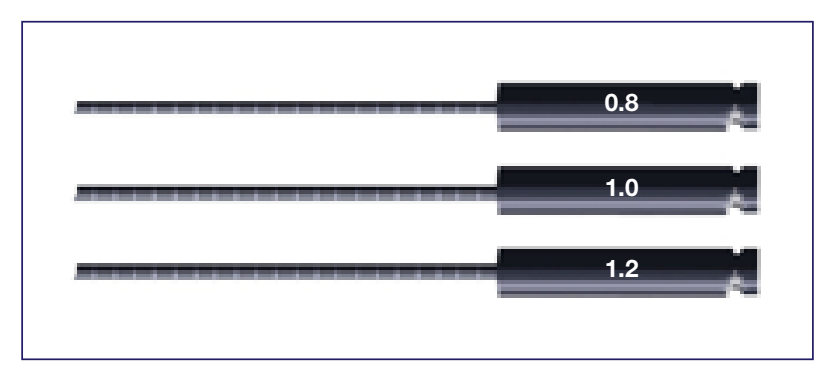

Figure 6. Trephine burs of the Zumax Broken Instrument Removal Kit (Zumax Medical) available in sizes 0.8, 1.0 and 1,2 $\mathrm{mm}$ in diameter.

The rest of the system comprises a stainless steel handle that can be fitted with either one of three extractors $(0.8,1.0$ and $1.2 \mathrm{~mm}$ in diameter) (Figure $7 \mathrm{~A}$ ) or a crab claw-shaped tweezer (Figure 7B). The crab claw-shaped tweezer is indicated for removal of fractured instruments that are visible directly on the pulp floor.

The extractors are microtubes fitted with a metal wedge inside that is pushed forward upon activation of a lever on a stainless steel handle (Figure $7 \mathrm{C}$ ) that can be attached to the extractors. The open lumen of the extractor is pushed over the exposed instrument head and the fragment is clamped by mechanically locking the instrument in the lumen of the extractor with the elongated metal wedge. The extractor and handle are carefully twisted clockwise and anticlockwise while exerting a pulling action on the file until it is retrieved.
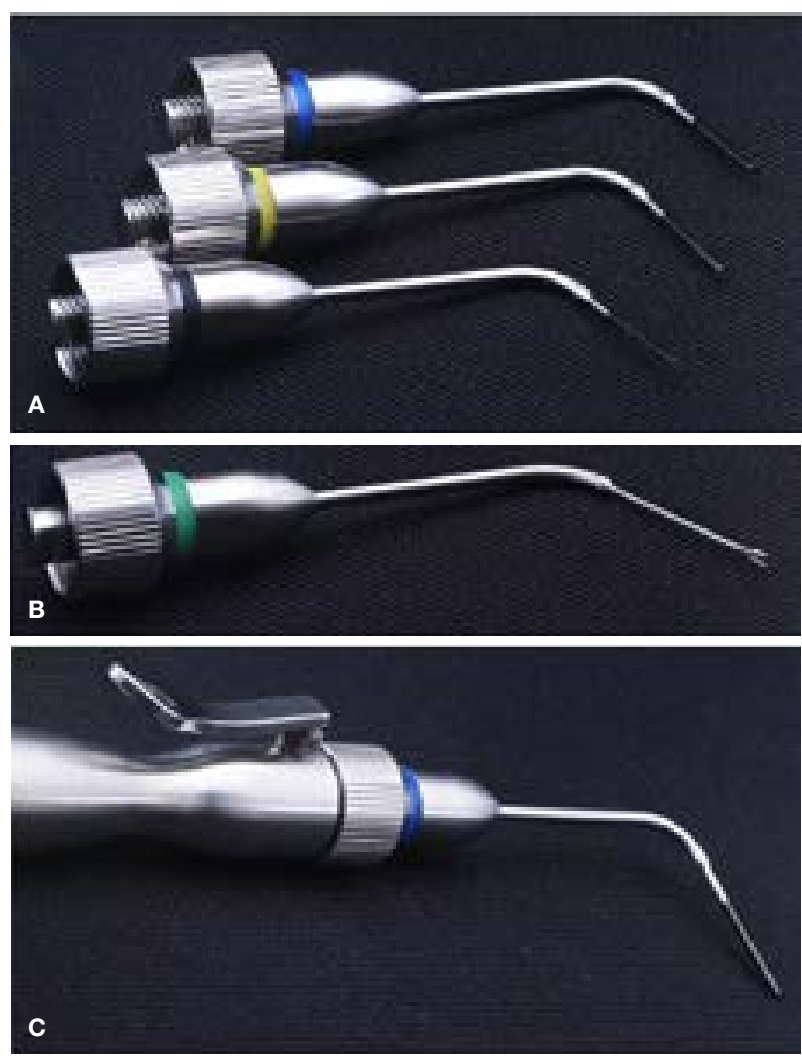

Figure 7.

A. Extractors $(0.8,1.0$ and $1.2 \mathrm{~mm}$ in diameter).

B. Crab claw-shaped tweezer.

C. The activation handle attached to one of the extractors of the Zumax Broken Instrument Removal Kit (Zumax Medical).

\section{CASE REPORT 4}

A 51-year-old female presented with a history of a fractured rotary file in the disto-buccal root canal system of her right maxillary first molar (Figure $8 \mathrm{~A}$ ). The tooth formed the distal abutment of a three-unit bridge extending from the right maxillary first premolar. Upon clinical examination it was noted that the abutment had decemented and it was decided to section the bridge before treatment.

Under high microscope magnification the coronal aspect of the fragment was visible. A no. 3 Start $X$ tip (Dentsply Sirona) (Figure 8B) was used to remove some restrictive dentine before an ultrasonic instrument (Endosonare file mounted in a U-File holder) was used around the coronal aspect of the tooth to create a trough of approximately $1.2 \mathrm{~mm}$ (Figure 8C). 
The $1 \mathrm{~mm}$ trephine was used to remove more dentine around the fractured segment to expose the coronal aspect of the fragment for about 2.5-3 mm (Figure 8D). The $1 \mathrm{~mm}$ Zumax extractor was pushed over the exposed instrument head and the fragment was secured by mechanically locking the instrument in the lumen of the extractor with the elongated metal wedge (Figure 8E).

The extractor and handle were carefully manipulated until the fragment was retrieved. Figure $8 \mathrm{~F}$ depicts the result after root canal preparation and obturation of the root canal system.

\section{iii. Terauchi File Retrieval Kit (TFRK)}

For the Terauchi File Retrieval Kit (TFRK) it is recommended to use no.2 and no. 3 GG burs (max 1000 rpm), ensuring minimal removal of the dentine to conserve the surrounding root structure. The canal should be enlarged to at least four sizes $(0.2 \mathrm{~mm})$ larger in diameter than the separated file. ${ }^{37}$ The no. 3 GG bur has a diameter of $0.9 \mathrm{~mm}$ and is the maximum $\mathrm{GG}$ bur to use for canal preparation. The use of larger GG burs increases the chances of stripped perforations, especially in curved canals.
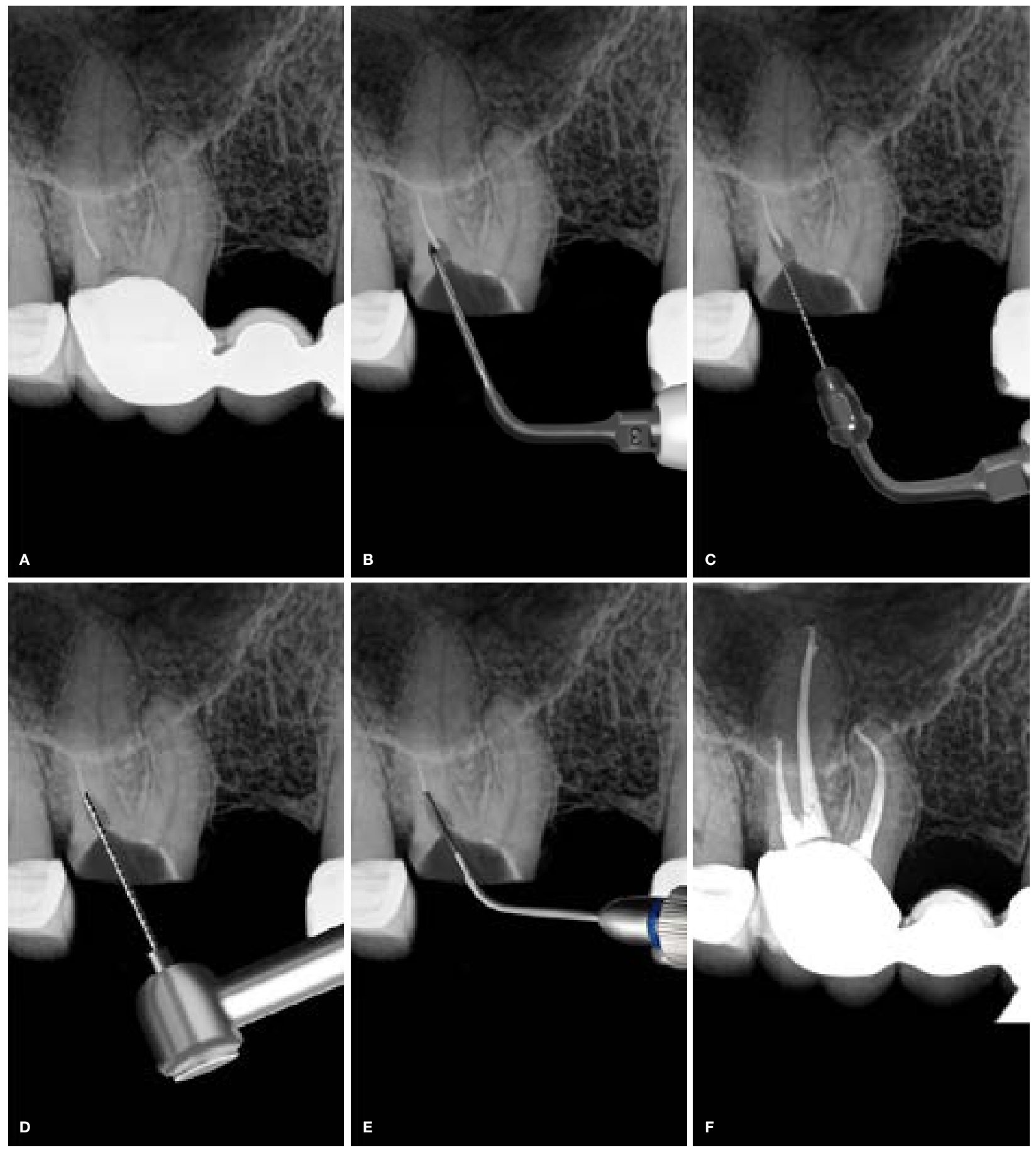

\section{Figure 8.}

A. Periapical radiograph of a maxillary left first molar showing a fractured file in the disto-buccal root canal.

B. Removal of restrictive dentine with Start X no. 3 tip.

D. Coronal aspect of fractured instrument exposed with a $1 \mathrm{~mm}$ trephine. E. Extractor placed over head of the fractured file in order to remove it.

C. Trough created around the coronal aspect of fractured file with an Endosonare file mounted in a U-File holder. 
The TFRK Micro-Trephine bur is used at $600 \mathrm{rpm}$, rotating in a counter-clockwise direction, to remove a small amount of dentine around the coronal aspect of the fractured segment (Figures 9A and B). It can also encourage a bound file segment to reverse-thread back coronally and loosen.

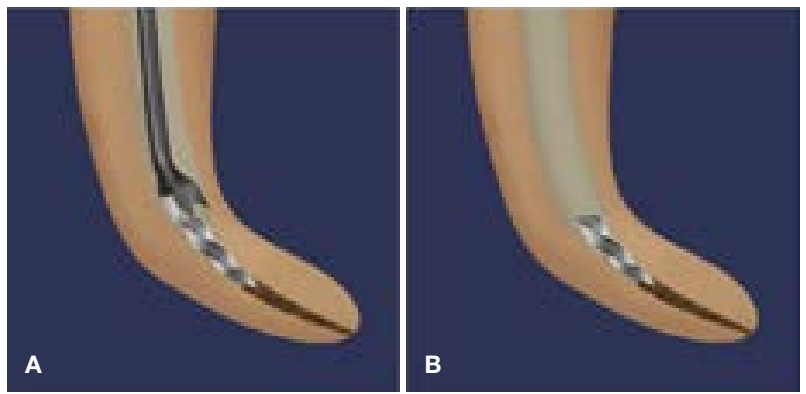

Figure 9. (Adapted from Terauchi, $2012^{37}$ )

A. TFRK Micro-Trephine bur is used to remove a small amount of dentine around the coronal aspect of the fractured segment.

B. The coronal aspect of the fractured segment is exposed.

An ultrasonic TFRK spear tip is brought into the canal and then activated on the dentine wall of the inner curvature to create a tiny space of approximately $1.0 \mathrm{~mm}$ away from the fractured surface of the file fragment (Figure 10A). The spear tips are extremely thin and sharp at the ends; it advisable to use the latch-grip rubber polishing point included in the TFRK to further thin and sharpen the spear tips before and between uses to improve their effectiveness and avoid removal of unnecessary tooth structure.

According to Buchanan ${ }^{38}$, it seems logical to trough the canal wall on the outside of its curvature, because that is where the fractured edge will be engaged. The problem is that, according to Terauchi ${ }^{37}$, troughing on the outside of the canal curvature does not work because: a) troughing the wall increases the curvature of the canal, while cutting the inside-of-the-curve canal wall straightens the canal, and b) activating the ultrasonic tip on the outside-of-the-curve wall hammers the segment and actually moves it further down the root canal.

After troughing the inner curvature, a shallow groove is cut along the outer curvature (Figure 10B) so that no obstruction can keep the fragment from being kicked out coronally by ultrasonic vibration. Finally, two specially designed microspoon tips are used to connect the inner and outer grooves that were created. The two microspoon tips face toward (the 6 o'clock tip) and away from (the 12 o'clock tip) the ultrasonic handpiece and are chosen relative to the direction of the canal curvature.

The root canal is then filled with EDTA solution to enhance the ultrasonic cavitation effect and acoustic streaming for removal. Ultrasonic vibration should be applied to the separated file in the space created between the fragment and the inner curve of the canal and move in "push and pull" motions until it is removed. Most separated files usually come out in 10-30 seconds with ultrasonics (Figure 10D). If a separated file shows resistance to disengagement for more than $60 \mathrm{sec}-$ onds, it is recommended to remove more dentine apically along the inside wall of the fragment before the next removal attempt.

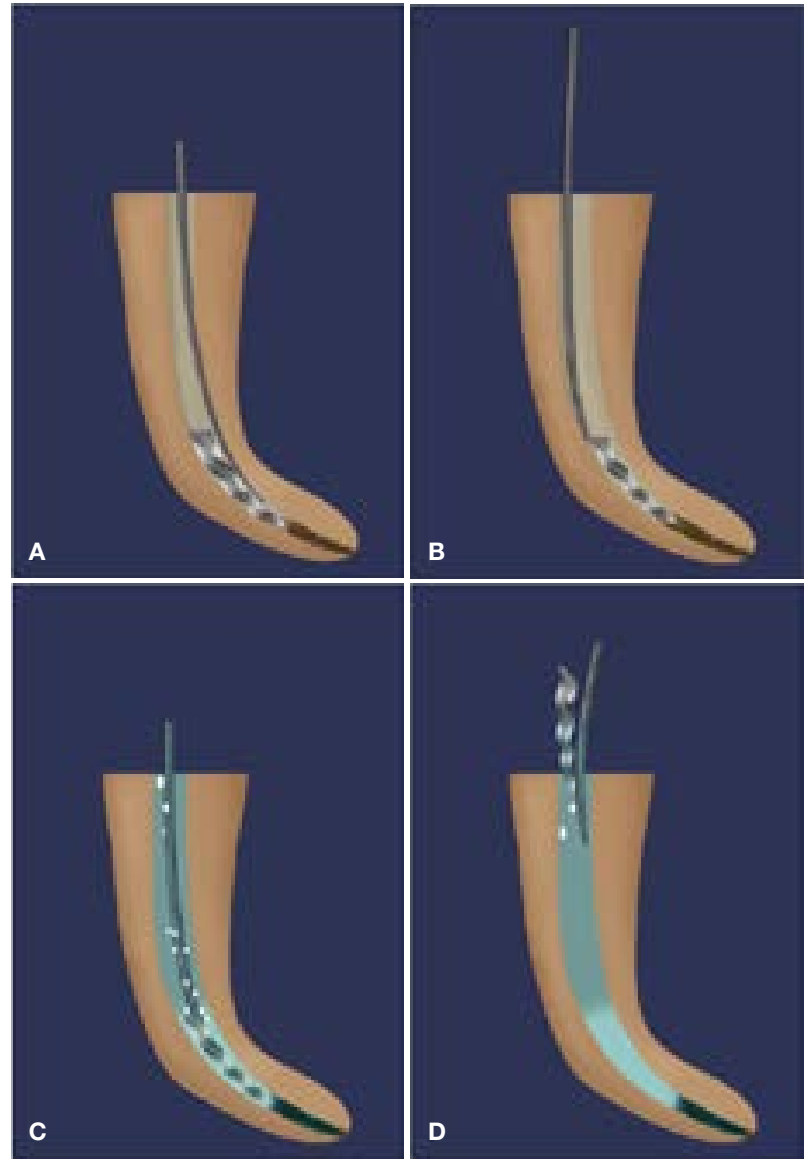

Figure 10. (Adapted from Terauchi, 2012 ${ }^{37}$ )

A. TFRK spear tip is activated on the inner curvature to create a tiny space of approximately $1.0 \mathrm{~mm}$ deep from the fractured surface of the file fragment.

B. A shallow groove is cut along the outer curvature.

C. The root canal is then filled with EDTA solution to enhance the ultrasonic cavitation effect and acoustic streaming for removal using the TFRK spear tip.

D. Separated file dislodged from the root canal.

All the ultrasonic tips in the TFRK are made of ductile stainless steel and it is possible to pre-bend them if necessary. The appropriate power setting when using these tips is typically in the lower quarter of the ultrasonic unit's power range and must be activated intermittently by tapping the foot control for one to two seconds, rather than being used in continuous mode. Intermittent activation keeps the tips from overheating and sends a relatively powerful ripple through the long, thin instruments. After two or three pulses, the tips are removed, cooled and cleaned with a wet alcohol sponge, and then replaced for further work until the file is loosened. Working with ultrasonic tips next to fractured file segments in a continuous rather than pulsed activation increases the risk of breaking the file segment into smaller pieces.

Terauchi ${ }^{37}$ demonstrates that separated files longer than $4.5 \mathrm{~mm}$ in any canal, or those in curved canals greater than $60^{\circ}$, are extremely difficult to retrieve with ultrasonic tips alone. On average, it took longer than nine minutes to remove fragments longer than $4.5 \mathrm{~mm}$, while separated files shorter than $4.5 \mathrm{~mm}$ were easy to retrieve with ultrasonics alone and the removal time was about five minutes. He concludes that ultrasonic removal attempts should be performed on a separated file shorter than $4.5 \mathrm{~mm}$ long, and ultrasonics in conjunction 
with another device, such as the loop device, should be considered if ultrasonic removal time exceeds five minutes. ${ }^{38,39}$

The Yoshi loop in the TFRK is a stainless steel microlasso that extends from the end of a stainless steel cannula attached to a handle with a retraction button for tightening the loop around a loosened file segment. If using the loop, the fragment must be loose and exposed peripherally by at least $0.7 \mathrm{~mm}$.

The Yoshi loop is prepared by moving the red retraction button forward to extend the wire lasso. A DG-16 explorer tip is then placed inside the lasso, and the retraction button carefully pulled backwards until the wire loop is felt to tighten on the explorer tine. This rounds the loop, leaving enough space to place it around the end of the file segment.

Before the explorer is removed from the loop, it is rotated back to a position parallel to the cannula to bend the rounded loop to a 45-degree angle. This rounded, angled loop wire is then ideally formed to drop down the root canal (Figure 11A) and around the coronal ex posed end of the file segment (Figure 11B), ideally seen under magnification.

The red retraction button is moved backwards to tighten the loop around the loosened file segment and is carefully tugged in several directions until the file is pulled out of the canal (Figure 11C).
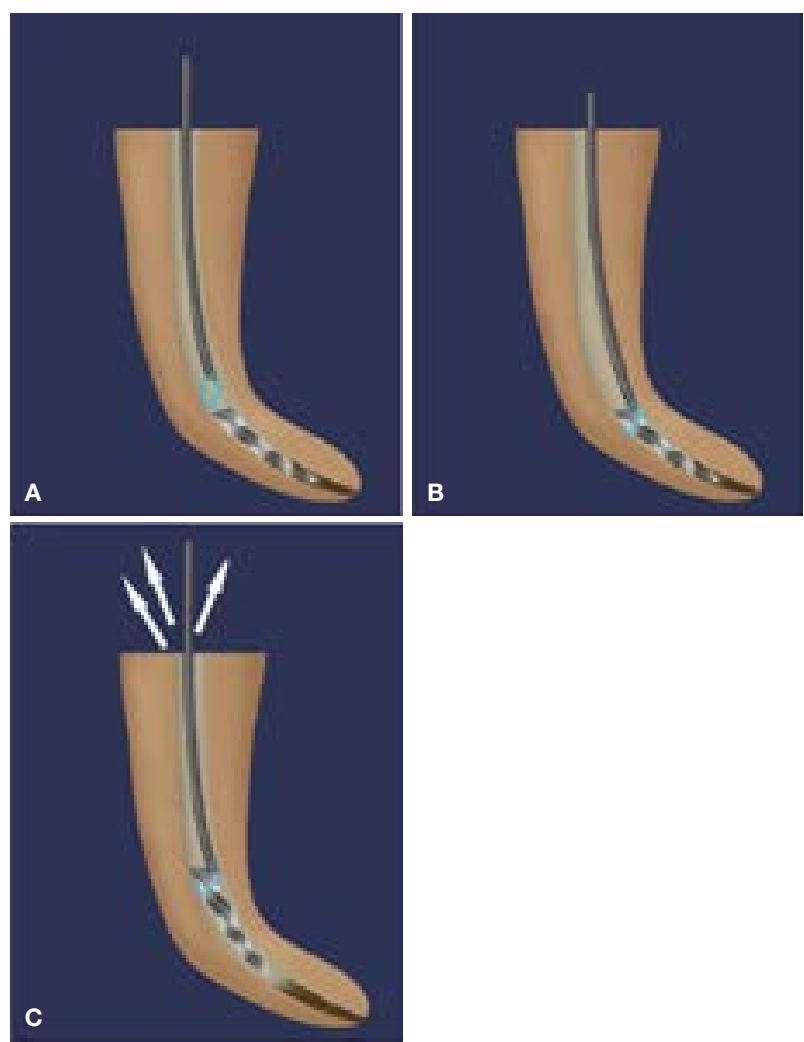

Figure 11. (Adapted from Terauchi, $2012^{37}$ )

A. Rounded, angled loop wire on the Yoshi loop dropped down the root canal under magnification

B. Loop wire is placed around the coronal exposed end of the file segment.

C. Retraction button on the handle is moved backwards to tighten the loop around the loosened file segment, before the handle is carefully tugged in several directions until the file is pulled out of the canal.

\section{CASE REPORT 5}

The patient, a 41-year-old male, presented with a poor root canal treatment and a fractured instrument in the mesio-buccal root canal of his left mandibular first molar (Figure 12A).

Number 2 and 3 GG burs were used to create a staging platform (Figure 12B) and a TFRK Micro-Trephine bur was used at $600 \mathrm{rpm}$ rotating in a counterclockwise direction to remove a small amount of dentine around the coronal aspect of the fractured segment (Figure 12C). An ultrasonically driven TFRK spear tip was brought into the canal and then activated on the dentine walls on the mesial and distal (Figure 12D) aspects of the fragment to create a space of approximately $2.5 \mathrm{~mm}$ deep from the fractured surface of the file fragment. The two microspoon tips were used to connect the inner and outer grooves created with the TFRK spear tip.

The root canal was filled with EDTA solution and ultrasonic vibration was applied to the separated file in the space created between the fragment and the canal walls. As the file could not be removed after 80 seconds and was more than $4.5 \mathrm{~mm}$ long, it was decided to use the Yoshi loop (Figure 12E) to retrieve it. Figures $12 \mathrm{~F}$ and $\mathrm{G}$ show, respectively, periapical radiographs of the tooth after file removal and the final obturation result after retreatment of the root canal systems.

\section{iv. EndoCowboy (Köhrer Medical Engineering))}

The EndoCowboy (Köhrer Medical Engineering) (Figure 13) is the latest endodontic instrument for removing broken root canal file segments from root canals. The separated instrument is also removed by placing a wire loop projecting from a needle (Figure 14) at the end of the EndoCowboy around the fractured end of the file, after which the loop can be tightened, securing it around the file segment to be removed. This micro-lasso tool can be adjusted precisely and makes it possible to grab files even deep down in the canal with minimally invasive access.

The technique is very similar to the Yoshi loop, but the wire of the lasso is (1) a lot stronger; (2) available in three different thicknesses; and (3) gives the clinician more confidence in removing fractured instruments. The manufacturer recommends a straight-line access to the separated instrument, which must be created with an ISO 70 file or a size 2 GG bur.

The head of the instrument should be exposed by creating a small circular space of at least $1 \mathrm{~mm}$ deep with an ultrasonic tip so that the lasso can be placed around the fractured instrument. The fragment can be loosened further with ultrasonics until a "dancing movement" of the fragment is observed. However, the authors have removed several ingrained instruments by exposing only the head before using the high-tear-resistant lasso wire to extract the file.

The EndoCowboy (Köhrer Medical Engineering) is held like a hand piece. After the preformed lasso is placed 

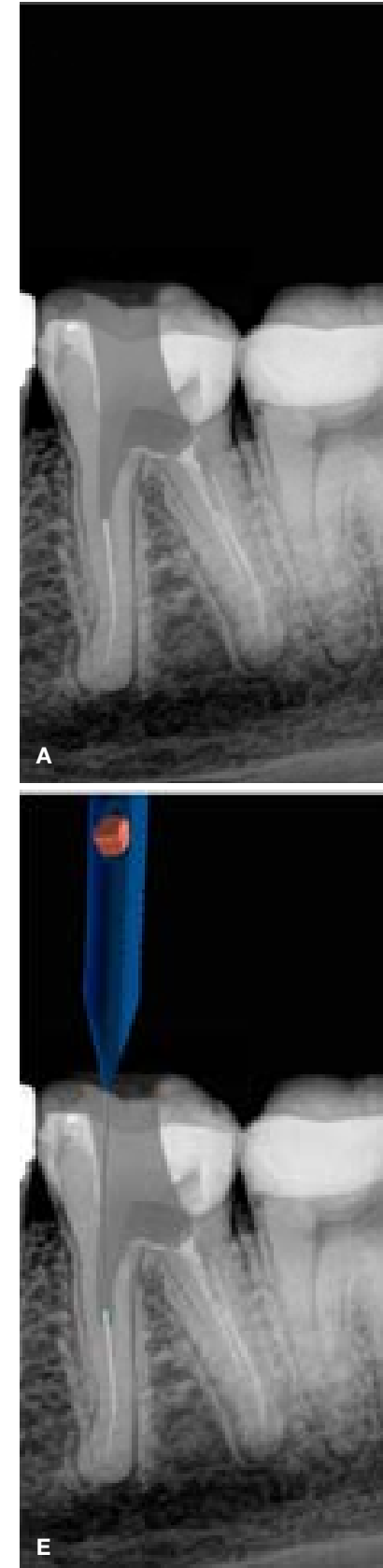

Figure 12.

A. Periapical radiograph of a mandibular left first molar showing a fractured file in the mesio-buccal root.

B. Staging platform created with a modified GG no. 3 and drill.

C. Coronal portion of the fracture file exposed using the TFRK microtrephine bur.

D. Space created around the fractured instrument both mesially and distally with an ultrasonically driven TFRK spear tip and spoon tip.

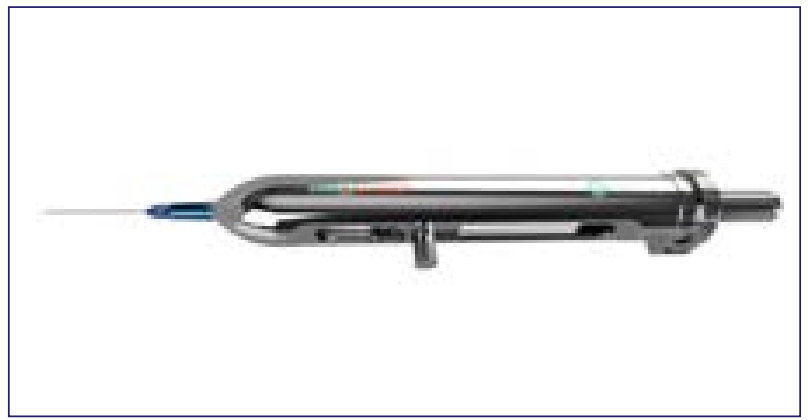

Figure 13. EndoCowboy (KÖhrer Dental).
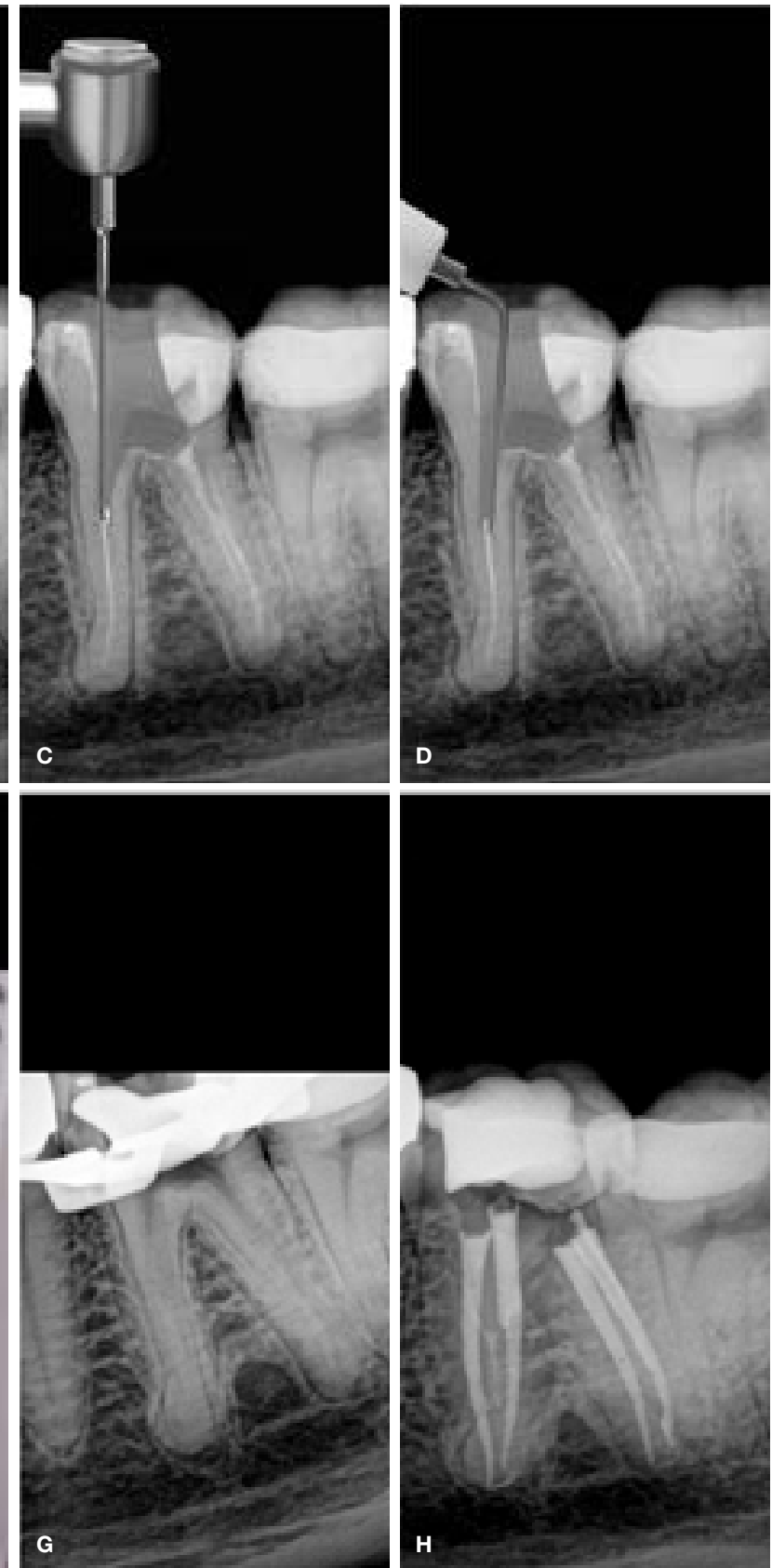

E. Yoshi loop placed around the exposed head of the fractured file. F. Extracted fractured fragment measuring $7 \mathrm{~mm}$ on a ruler.

G. Periapical radiograph showing successful removal of fractured instrument.

H. Postoperative obturation result.

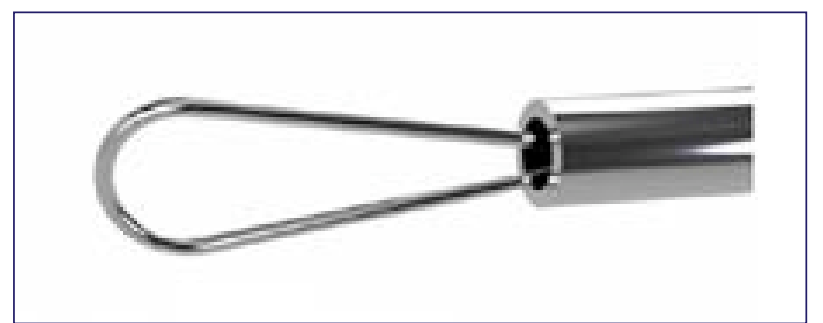

Figure 14. Magnified view of the wire loop projecting from a needle cannula. 
around the separated instrument, it can be closed by tightening the wire. One of the unique characteristics of the EndoCowboy (Köhrer Medical Engineering) is that the dental assistant can help to operate the device. This person can turn the adjusting wheel clockwise to control the tension of the lasso. This allows the doctor to concentrate fully on placing the lasso around the separated instrument without using any hand movements to close the loop around the instrument.

In order to exert enough tension on the separated instrument, the lasso must be pulled tightly around it. The device is fitted with a special built-in ball bearing that allows precise tightening and maximum tactile feedback of the wire loop tension when the dental assistant adjusts the wheel. Once the required amount of tension is set the lasso will remain in the desired position, enabling the doctor to concentrate on the movement need to extract the file. However, too much tension on the lasso must be avoided, since it can cause the wire to tear. The fractured file is extracted from the root canal using a movement similar to extracting a tooth.

As mentioned before, the EndoCowboy specially drawn stainless steel lasso guarantees maximum tear resistance to enable strong traction. The wires are available in three different thicknesses preloaded in metal cannulas. The thinnest wire is $0.8 \mathrm{~mm}$ preloaded in a cannula of $0.3 \mathrm{~mm}$; a standard $0.1 \mathrm{~mm}$ wire is loaded in a $0.4 \mathrm{~mm}$ cannula and a thicker wire of $0.12 \mathrm{~mm}$ in a $0.5 \mathrm{~mm}$ cannula.

\section{CASE REPORT 6}

A 54-year-old male presented with bite sensitivity and occasional discomfort on his right mandibular first molar (Figure 16A). Radiographic examination revealed a poor root canal treatment with a fractured file in the mesiobuccal root canal system and the possibility of a missed root canal system in the distal root.

An access cavity was prepared trough the existing crown and a no. 3 GG bur was used to create a staging platform. A size 15 Endosonare file (Dentsply Sirona) mounted in a U-File holder (Endo Kit E12, NSK) was used to trough around the coronal aspect of the fragent (Figure 16B), before a TFRK spear tip was used to create a further space of approximately $1.5 \mathrm{~mm}$ deep on the dentine wall of the inner curvature from the fractured surface of the file fragment. The EndoCowboy, preloaded with the a standard $0.1 \mathrm{~mm}$ wire in a $0.4 \mathrm{~mm}$ cannula, was introduced into the root canal, the preformed lasso was positioned around the separated instrument, and the lasso closed by tightening the wire by turning the adjusting wheel clockwise (Figure 16C). The fractured fragment was extracted from the root canal using a pulling action. Figure $16 \mathrm{~d}$ shows the final obturation result after retreatment of all the root canal systems.

\section{CONCLUSION}

Procedural errors, of which instrument fracture is probably the most challenging to manage, can occur during any stage of root canal cleaning and shaping.
Instrument fracture is likely to have a negative impact on the long-term prognosis of the tooth. Proper training and clinical experience, together with adherence to sound clinical principles and guidelines for clinical use, can limit the incidence of instrument separation. ${ }^{40}$ When faced with a separated endodontic instrument the clinician has the option to bypass it, to leave it in situ or to retrieve it.

Removal of fractured instruments is influenced by the length of the instrument, its diameter and position as well as the root canal anatomy and curvature. ${ }^{41} \mathrm{~A}$ study by Wilcox et al. ${ }^{42}$ demonstrates that the least possible dentine structure should be sacrificed for file removal attempts, as it has been reported that canal enlargement of $40 \%$ to $50 \%$ of the root width increases susceptibility to vertical fracture. Suter et al. ${ }^{7}$ recommend that attempts to remove fractured instruments from root
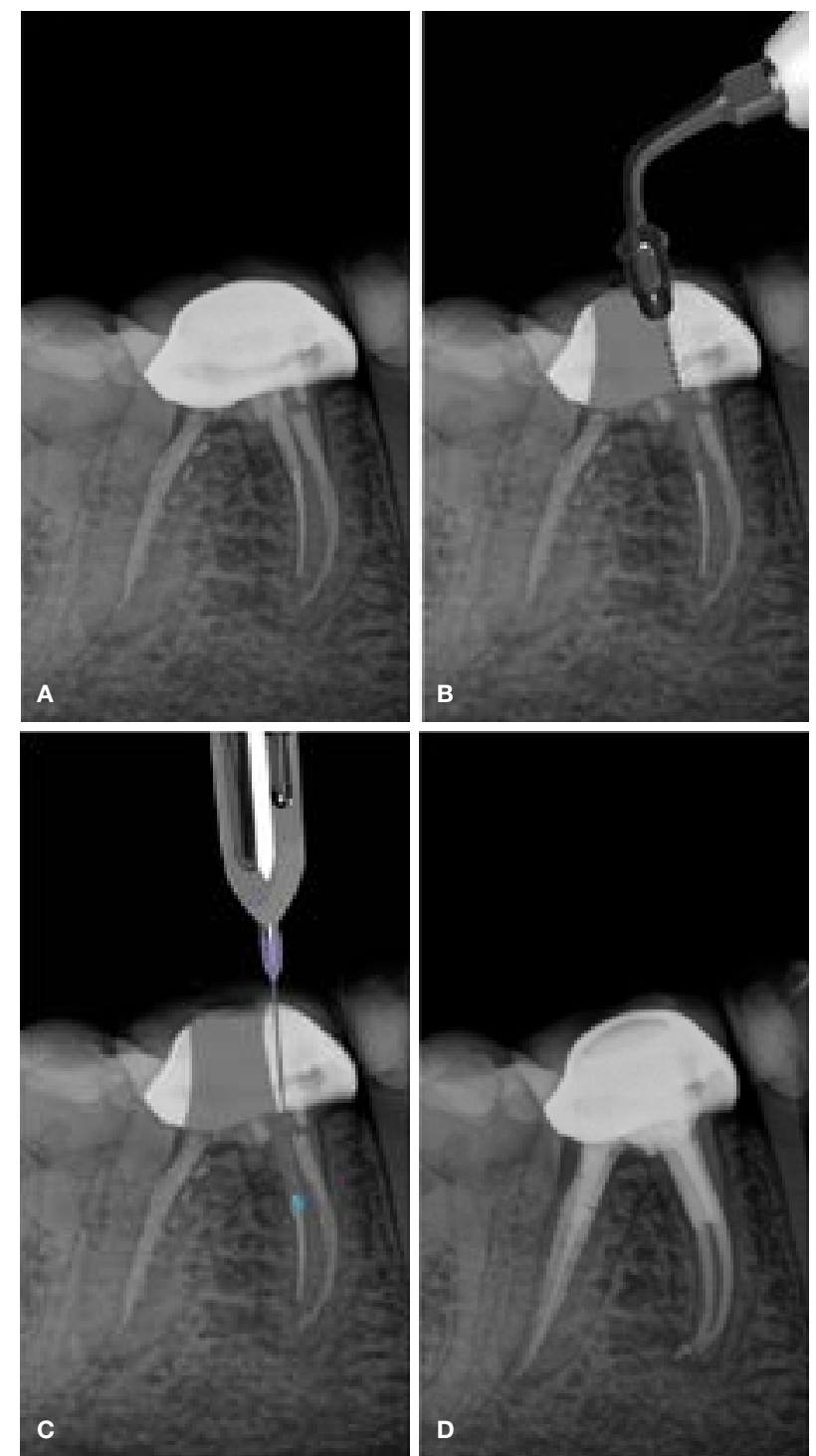

Figure 16.

A. Pre-operative periapical radiograph of a mandibular right first molar with file fracture in the mesio-buccal root canal.

B. A size 15 Endosonare file mounted in a U-File holder to trough around the coronal aspect of the fragment.

C. The EndoCowboy positioned around the separated instrument in order to extract the fragment.

D. Postoperative periapical radiograph after retreatment of all the root canal systems. 
canals should not exceed 45 to 60 minutes because of operator fatigue or overenlargement of the root canals. The extended removal time may also lead to iatrogenic errors such as root perforation and vertical root fractures. In this paper the authors illustrate different approaches to the clinical management of endodontic instrument fracture, with specific focus on file retrieval methods and commercially available retrieval systems. The case studies presented should provide the clinician with helpful insights into the clinical management of these often challenging scenarios.

\section{References}

1. Iqbal $A$. The factors responsible for endodontic treatment failure in the permanent dentitions of the patients reported to the college of dentistry, the University of Aljouf, Kingdom of Saudi Arabia. JCDR. 2016; 10(5): ZC146.

2. Sjögren $U$, Hägglund $B$, Sundqvist $G$, Wing $K$. Factors affecting the long-term results of endodontic treatment. J Endod. 1990; 16(10): 498-504.

3. Siqueira Jr JF. Aetiology of root canal treatment failure: why well-treated teeth can fail. Int. Endod. J. 2001; 34(1): 1-10.

4. McGuigan M, Louca C, Duncan H. Clinical decision-making after endodontic instrument fracture. Br. Dent. J. 2013; 214(8): 395.

5. Grossman LI. Guidelines for the prevention of fracture of root canal instruments. Oral Surg,, Oral Med, Oral Pathol, Oral Radiol. 1969; 28(5): 746-52.

6. Parashos P, Gordon I, Messer HH. Factors influencing defects of rotary nickel-titanium endodontic instruments after clinical use. J Endod. 2004; 30(10): 722-5.

7. Suter B, Lussi A, Sequeira P. Probability of removing fractured instruments from root canals. Int. Endod. J. 2005; 38(2): 112-23.

8. McLeod N, Davies B, Brennan P. Bisphosphonate osteonecrosis of the jaws: an increasing problem for the dental practitioner. Br Dent J. 2007; 203(11): 641.

9. Saunders JL, Eleazer PD, Zhang P, Michalek S. Effect of a separated instrument on bacterial penetration of obturated root canals. J Endod. 2004; 30(3): 177-9.

10. De Chevigny C, Dao TT, Basrani BR, et al. Treatment outcome in endodontics: the Toronto study - phase 4: initial treatment. J Endod. 2008; 34(3): 258-63.

11. Parashos P, Messer HH. Rotary NiTi instrument fracture and its consequences. J Endod. 2006; 32(11): 1031-43.

12. Torabinejad M, Lemon RR. Procedural accidents. Principles and practice of endodontics. 2002; 2: 306-23.

13. Frota LMA, Aguiar BA, Aragão MGB, De Vasconcelos BC. Removal of separated endodontic K-File with the aid of hypodermic needle and cyanoacrylate. Case reports in Dentistry. 2016.

14. Hülsmann M. Removal of fractured instruments using a combined automated/ultrasonic technique. J. Endod. 1994; 20(3): 144-6.

15. Gencoglu N, Helvacioglu D. Comparison of the different techniques to remove fractured endodontic instruments from root canal systems. Eur J Dent. 2009; 3(02): 90-5.

16. Shiyakov KK, Vasileva RI. Success for removing or bypassing instruments fractured beyond the root canal curve - 45 clinical cases. JIMAB - Annual Proceedings Scientific Papers. 2014; 20(3): 567-71.

17. Nevares G, Cunha RS, Zuolo ML, Da Silveira Bueno CE. Success rates for removing or bypassing fractured instruments: a prospective clinical study. J Endod. 2012; 38(4): 442-4.

18. Iqbal MK. Nonsurgical ultrasonic endodontic instruments. Dental Clinics. 2004; 48(1): 19-34.

19. Cohen S, Burns R. Pathways of the pulp, $8^{\text {th }}$ edn. St Louis, MO: Mosby; 2002.

20. Ruddle CJ. Nonsurgical retreatment. J. Endod. 2004; 30(12): $827-45$.
21. Ward JR, Parashos P, Messer HH. Evaluation of an ultrasonic technique to remove fractured rotary nickel-titanium endodontic instruments from root canals: an experimental study. J. Endod. 2003; 29(11): 756-63.

22. Ruddle CJ. Broken instrument removal: the endodontic challenge. Dent Today. 2002; 21(7): 70-81.

23. Nagai O, Tani N, Kayaba Y, Kodama S, Osada T. Ultrasonic removal of broken instruments in root canals. Int Endod J. 1986; 19(6): 298-304.

24. Cujé J, Bargholz C, Hülsmann M. The outcome of retained instrument removal in a specialist practice. Int Endod J. 2010; 43(7): 545-54.

25. Fu M, Zhang Z, Hou B. Removal of broken files from root canals by using ultrasonic techniques combined with dental microscope: a retrospective analysis of treatment outcome. J Endod. 2011; 37(5): 619-22.

26. Ward JR, Parashos P, Messer HH. Evaluation of an ultrasonic technique to remove fractured rotary nickel-titanium endodontic instruments from root canals: clinical cases. J Endod. 2003; 29(11): 764-7.

27. Hülsmann M, Schinkel I. Influence of several factors on the success or failure of removal of fractured instruments from the root canal. Dent. Traumatol. 1999; 15(6): 252-8.

28. Ruddle C. Nonsurgical endodontic retreatment. In Cohen S, Burns RC editors: Pathways of the pulp, Mosby, St. Louis MO. 2002; 875-929.

29. Shen Y, Peng B, Cheung GS-p. Factors associated with the removal of fractured NiTi instruments from root canal systems. Oral Surg, Oral Med, Oral Pathol, Oral Radiol, Endod. 2004; 98(5): 605-10.

30. Dominici JT, Clark S, Scheetz J, Eleazer PD. Analysis of heat generation using ultrasonic vibration for post removal. J Endod. 2005; 31(4): 301-3.

31. Gluskin AH, Ruddle CJ, Zinman EJ. Thermal injury through intraradicular heat transfer using ultrasonic devices: precautions and practical preventive strategies. J Am Dent Assoc. 2005; 136(9): 1286-93.

32. Hashem AAR. Ultrasonic vibration: temperature rise on external root surface during broken instrument removal. J Endod. 2007; 33(9): 1070-3.

33. Sweatman TL, Baumgartner JC, Sakaguchi RL. Radicular temperatures associated with thermoplasticized gutta-percha. J Endod. 2001; 27(8): 512-5.

34. Souter NJ, Messer HH. Complications associated with fractured file removal using an ultrasonic technique. J Endod. 2005; 31(6): 450-2.

35. Marsicovetere ES, Clement DJ, Carlos E. Morphometric video analysis of the engine-driven nickel-titanium Lightspeed instrument system. J Endod. 1996; 22(5): 231-5.

36. Madarati AA, Qualtrough AJ, Watts DC. Efficiency of a newly designed ultrasonic unit and tips in reducing temperature rise on root surface during the removal of fractured files. J Endod. 2009; 35(6): 896-9.

37. Terauchi Y. Separated file removal. Dent. Today. 2012; 31(5): 108-10.

38. Buchanan, LS. Removing separated files with the Terauchi file retreival kit. Roots. 2016; 1: 20 -3.

39. Terauchi Y, O'Leary L, Kikuchi I, et al. Evaluation of the efficiency of a new file removal system in comparison with two conventional systems. J Endod. 2007; 33(5): 585-8.

40. Alapati SB, Brantley WA, Svec TA, Powers JM, Nusstein JM, Daehn GS. SEM observations of nickel-titanium rotary endodontic instruments that fractured during clinical use. J Endod. 2005; 31(1): 40-3.

41. Alomairy $\mathrm{KH}$. Evaluating two techniques on removal of fractured rotary nickel-titanium endodontic instruments from root canals: an in vitro study. J Endod. 2009; 35(4): 559-62.

42. Wilcox LR, Roskelley C, Sutton T. The relationship of root canal enlargement to finger-spreader induced vertical root fracture. J Endod. 1997; 23(8): 533-4. 